\title{
International conference: Modeling \& Design of Molecular Materials - MDMM 2008 (23-28 June 2008, Piechowice, Poland)
}

\author{
W. Andrzej Sokalski • Jarosław V. Burda • \\ Jerzy Leszczyński
}

Published online: 24 March 2009

(C) Springer-Verlag 2009

The MDMM 2008 meeting was devoted to discussions and presentations of current computational methods and their applications in molecular modeling, design of molecular materials, and related fields. This conference continues a tradition of alternating Polish-Czech (+American) meetings that have taken place in the Czech Republic and Poland since 2003. The MDMM2008 meeting in Piechowice, Poland included the following sessions: advances in computational methods, designed biomaterials, drug design, interactions in molecular materials, modeling biomaterials, zeolites, chemical reactions, nucleic acids, protein dynamics, and structure prediction (for more details see http://mml.ch.pwr.wroc.pl/ workshop/2008/).

MDMM2008 speakers included among others $\mathrm{T}$. Wesołowski (Geneva, Switzerland), M. Meuwly (Basel, Switzerland), R. van Santen (Eindhoven, The Netherlands), J. Sauer (Berlin, Germany), P. Boulet (Marseille, France), B. Szyja (Eindhoven, The Netherlands), J. S. Murray (Cleveland, OH, USA), H. Cheng (Air Products \& Materials Inc., Allentown, PA, USA), O. Shishkin (Kharkov, Ukraine), A. Toro-Labbe (Santiago, Chile), P. Politzer (New Orleans, LA,
USA), P. Paneth (Łódź, Poland), T. Clark (Erlangen, Germany), A. Koliński (Warsaw, Poland), A. Liwo (Gdańsk, Poland), R.A. Poirier (St.John's, Canada), A. Tachibana (Kyoto, Japan), Z. Latajka (Wrocław, Poland), J. C. Wojdeł (Barcelona, Spain), M. Ramos (Porto, Portugal), T. Brinck (Stockholm, Sweden), J. Koca (Brno, Czech Republic), P. Kozłowski (Louisville, KY, USA), P. Tchounwou (Jackson, USA), S. Guccione (Catania, Italy), J. Burda (Prague, Czech Republic), L. Berlicki (Wroclaw, Poland), T. Zimmermann (Praha, Czech Republic), E. Muratov (Odessa, Ukraine), P. Hobza (Prague, Czech Republic), J. Rak (Gdansk, Poland), J. Šponer (Brno, Czech Republic), P. Cysewski (Bydgoszcz, Poland), J. Leszczyński (Jackson, MS, USA). One session, which was sponsored by Fujitsu Poland, was devoted to commercial tools for computational chemistry. In addition, 53 posters were presented during two afternoon poster sessions.

The 25 articles published in this volume highlight the wide variety of contributions to the conference. We would like to express our sincere gratitude to all the authors for their contributions.

W. A. Sokalski $(\bowtie)$

Institute of Physical \& Theoretical Chemistry,

Wrocław University of Technology Wyb,

Wyspianskiego 27,

50-370 Wrocław, Poland

e-mail: sokalski@pwr.wroc.pl

J. V. Burda

Faculty of Mathematics \& Physics, Charles University,

Ke Karlovu 3,

12116 Prague, Czech Republic

J. Leszczyński

Interdisciplinary Center for Nanotoxicity,

Jackson State University,

1325 J.R. Lynch Street,

Jackson, MS 39217-0510, USA 